\title{
Robust Controller Design: A Bounded-Input Bounded-Output Worst-Case Approach
}

\author{
Munther A. Dahleh* \\ Department of Electrical Engineering and Computer Science \\ Massachuasetts Institute of Technology \\ Cambridge, MA 02139
}

\begin{abstract}
This chapter introduces a general framework for designing robust control systems in the presence of uncertainty when the specifications are posed in the time-domain. Necessary and sufficient conditions for performance robustness and a synthesis methodology utiliaing linear programming are presented. Since many of the past work involved quadratic performance objectives, this chapter outlines a set of new tools needed to handle Peak-to-Peak specifications. The objective in here is to present many of the central results in this discipline in an intuitive fashion rather than a complete rigorous treatment of the subjeet.
\end{abstract}

-This research was supported by Wright Patterson AFB under grant F33615-90-C:-3608. C'.S. Draper Laboratory under grant DL-H-441636 and NSF under grant 9157306-ECS. 


\section{Introduction}

Feedback controller design is primarily concerned with designing control systems that can deliver high quality performance despite the presence of uncertainty. Eventhough a real system is not uncertain, it is desirable to think of it as such to reflect our imprecise or partial knowledge of its dynamics. On the other hand, uncertainty in the noise and disturbances can be cast under "real uncertainties", as it is practically impossible to provide exact models of such inputs. The description of this uncertainty, plant or input uncertainty, depends on the particular physical system and the operation environment and thus it is problem-dependent. To aid the designer in this process, analytical theory has been developed to provide a design methodology for synthesizing controllers to achieve "certain" performance objectives in the presence of a specific class of uncertainty. It is understood that a particular design problem may not fit the assumptions needed in any of these problems, nevertheless such methods can provide at least three important pieces of information to the designer (one can think of many more): The first is providing a starting point for design, maybe in terms of achieving robust stability and nominal performance, but possibly also achieving robust performance. The second is highlighting the tradeoffs in designing the controller for the system given. As intuitively expected, robustifying the system to handle larger classes of uncertainty results in more conservative design and hence in the loss of some performance. The third is capturing in a quantitative way the fundamental limitations and capabilities of feedback design. This is possibly the most important of all since one would like to know whether certain performance specifications can be achieved, and if so, with what controllers. Examples of such analytical theory are the $H_{2}, H_{\infty}$ and the $\ell_{1}$ theory.

The $\ell_{1}$ theory is primarily a time-domain theory in which performance specifications as well as input and plant uncertainty are posed in the timedomain in terms of the maximum norm $\left(\ell_{\infty}\right.$-norm). This is a natural setup since most performance specifications are magnitude-specifications such 
as tracking errors, maximum deviations from a nominal point, limits on actuator authority and so on. Disturbances in general have the property of persistence and boundedness and are best described in terms of the $\ell_{\infty}$ norm. Examples of such disturbances are wind gusts, friction and so on.

In this chapter, an overview of the $\ell_{1}$ theory is given starting from simple nominal performance problems and leading all the way to a general theory that allows for synthesizing controllers to achieve robust performance. The basic ideas involved in concepts of robust stabilization are introduced in a simple intuitive way, without obscuring these ideas through rigorous proofs. The synthesis of $\ell_{1}$ controllers is explained through an analogy with standard linear programs, the difference being only in that the $\ell_{1}$ problem has an infinite number of variables. Most of the exact proofs are referred to as they are needed.

\section{Preliminaries}

First, some notation regarding standard concepts for input/output systems. For more details, consult $[1,2]$ and references therein.

$\ell_{\infty, e}$ denotes the extended space of sequences in $\mathbb{R}^{N}, f=\left\{f_{0}, f_{1}, f_{2}, \ldots\right\}$. $\ell_{\infty}$ denotes the set of all $f \in \ell_{\infty, e}$ such that

$$
\|f\|_{\ell_{\infty}}=\sup _{k}|f(k)|_{\infty}<\infty
$$

where $|f(k)|_{\infty}$ is the standard $\ell_{\infty}$ norm on vectors. $\ell_{\infty, e} \backslash \ell_{\infty}$ denotes the set $\left\{f: f \in \ell_{\infty, e}\right.$ and $\left.f \notin \ell_{\infty}\right\} . \ell_{p}, p \in[1, \infty)$, denotes the set of all sequences, $f=\left\{f_{0}, f_{1}, f_{2}, \ldots\right\}$ in $R^{N}$ such that

$$
\|f\|_{\ell_{p}}=\left(\sum_{k}|f(k)|_{p}^{p}\right)^{1 / p}<\infty .
$$

$c_{0}$ denotes the subspace of $\ell_{\infty}$ in which every function $x$ satisfies

$$
\lim _{k \rightarrow \infty} x(k)=0
$$

$S$ denotes the standard shift operator. 
$P_{k}$ denotes the $k^{\text {th }}$-truncation operator on $\ell_{\infty, e}$ :

$$
P_{k}:\left\{f_{0}, f_{1}, f_{2}, \ldots\right\} \rightarrow\left\{f_{0}, \ldots, f_{k}, 0, \ldots\right\}
$$

Let $H: \ell_{\infty, e} \longrightarrow \ell_{\infty, e}$ be a nonlinear operator. $H$ is called causal if

$$
P_{k} H f=P_{k} H P_{k} f, \quad \forall k=0,1,2, \ldots,
$$

$H$ is called strictly causal if

$$
P_{k} H f=P_{k} H P_{k-1} f, \quad \forall k=0,1,2, \ldots
$$

$H$ is called time-invariant if it commutes with the shift operator:

$$
H S=S H .
$$

Finally, $H$ is called $\ell_{p}$ stable if

$$
\|H\|=\sup _{k} \sup _{\substack{f \in \ell_{p}, e \\ P_{k} f \neq 0}} \frac{\left\|P_{k} H f\right\| l_{p}}{\left\|P_{k} f\right\| \ell_{p}}<\infty .
$$

The quantity $\|H\|$ is called the induced operator norm over $\ell_{p}$.

$\mathcal{L}_{T V}$ denotes the set of all linear causal $\ell_{\infty}$-stable operators. This space is characterized by infinite block lower triangular matrices of the form:

$$
\left(\begin{array}{ccc}
H_{00} & & 0 \\
H_{10} & H_{11} & \\
\vdots & \vdots & \ddots
\end{array}\right)
$$

where $H_{i j}$ is a $p \times q$ matrix. This infinite matrix representation of $H$ acts on elements of $\ell_{\infty}^{q}$ by multiplication, i.e. if $u \in \ell_{\infty}^{q}$, then $y:=H u \in \ell_{\infty}^{p}$ where $y(k)=\sum_{j=0}^{k} H_{k j} u(j) \in \mathbb{R}^{p}$. The induced norm of such an operator is given by:

$$
\|H\|_{\mathcal{L}_{T V}}=\sup _{i}\left|\left(H_{i 1} \ldots H_{i i}\right)\right|_{1}
$$

where $|A|_{1}=\max _{i} \sum_{j}\left|a_{i j}\right| . \mathcal{L}_{T I}$ denotes the set of all $H \in \mathcal{L}_{T V}$ which are time-invariant. It is well known that $\mathcal{L}_{T I}$ is isomorphic to $\ell_{1}$ and the matrix representation of the operator has a Toeplitz structure. Every element in $\mathcal{L}_{T I}$ is associated with a $\lambda$-transform defined as:

$$
\hat{H}(\lambda)=\sum_{k=0}^{\infty} H(k) \lambda^{k}
$$


The collection of all such transforms is usually denoted by $\mathbf{A}$, which will be equipped with the same norm as the $\ell_{1}$ norm.

Throughout this paper, systems are thought of as operators. So, the composition of two operators $G, H$ is denoted as $G H$, if both are time-invariant then $G H \in \ell_{1}$ (or $\mathcal{L}_{T I}$ ), and the induced norm is denoted by $\|G H\|_{1}$. When the $\lambda$-transform is referred to specifically, we use the notation $\hat{H}$ for the transform of $H$. Also, all operator spaces are matrix-valued functions whose dimensions will be suppressed in general whenever understood from the context.

\section{The $\ell_{1}$ Norm}

Let $T$ be a linear time-invariant system given by

$$
z(t)=(T w)(t)=\sum_{k=0}^{t} T(k) w(t-k) .
$$

The inputs and outputs of the system are measured by their maximum amplitude over all time; otherwise known as the $\ell_{\infty}$ norm, i.e.

$$
\|w\|_{\infty}=\max _{j} \sup _{k}\left|w_{j}(k)\right| \text {. }
$$

The $\ell_{1}$ norm of the system $T$ is precisely equal to the maximum amplification the system exerts on bounded inputs. This measure defined on the system $T$ is known as the induced operator norm and is mathematically defined as follows:

$$
\|T\|=\sup _{\|w\|_{\infty} \leq 1}\|T w\|_{\infty}=\|T\|_{1}
$$

where $\|T\|_{1}$ is the $\ell_{1}$-norm of the pulse response and is given by

$$
\|T\|_{1}=\max _{i} \sum_{j} \sum_{k}\left|t_{i j}(k)\right|
$$

A system is said to be $\ell_{\infty}$ - stable if it has a bounded $\ell_{1}$ norm and the space of all such system will be denoted by $\ell_{1}$. From this definition, it is clear that the system attenuates inputs if its $\ell_{1}$ norm is strictly less than unity. For control systems applications, if the objective involves minimizing 
maximum deviations, rejection of bounded disturbances, tracking uniformly in time, or meeting certain objectives with hard constraints on the signals, a problem with an $\ell_{1}$ norm criterion arises.

In the case where the inputs and outputs of the linear system are measured by the $\ell_{2}$ norm, then the gain of the system is given by the $H_{\infty}$ norm and is given by:

$$
\|\hat{T}\|_{\infty}=\sigma_{\max }\left(\hat{T}\left(e^{i \theta}\right)\right) .
$$

The two induced norms are related as follows:

$$
\|\hat{T}\|_{\infty} \leq C\|T\|_{1}
$$

where $C$ is a constant depending only on the dimension of the matrix $T$. In other words, every system inside $\ell_{1}$ is also inside $H_{\infty}$, however the converse is not true. This means that there exists $\ell_{2}$ stable linear time-invariant systems that are not $\ell_{\infty}$ stable; an example is the function with the $\lambda$-transform given by $[3]$;

$$
\hat{T}(\lambda)=e^{\frac{1}{1-\lambda}} .
$$

Thus, for LTI systems, minimizing the $\ell_{1}$ norm of a systems guarantees that the $H_{\infty}$ norm is bounded. This means that this system will have good $\ell_{2}$-disturbance rejection properties as well as $\ell_{\infty}$-disturbance rejection properties. Also, the $\ell_{1}$ norm is more tied-up to BIBO stability notions and hence quite desirable to work with. The disadvantage in working with the $\ell_{1}$ norm is the fact that it is a Banach space of operators operating on a Banach space, not a Hilbert space. Many of the standard tools are not usable, however, this overview will present new techniques for handling problems of this kind.

\section{Stability and Parameterization}

Consider the system $G$ described as in Figure $1 . G$ is a $2 \times 2$ Block matrix, $u$ is the control input, $y$ is the measured output, $w$ is the exogenous input, $z$ is the regulated output, and $K$ is the feedback controller. Let $H(G, K)$ 


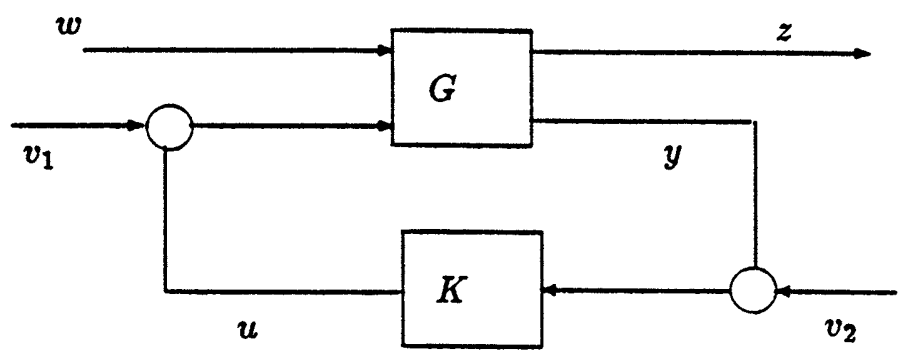

Figure 1: Closed Loop System

denote the map

$$
H(G, K)=\left(\begin{array}{c}
w \\
v_{1} \\
v_{2}
\end{array}\right), \longrightarrow\left(\begin{array}{c}
z \\
u \\
y
\end{array}\right)
$$

The closed loop system is $\ell_{\infty}$-stable if and only if $H(G, K) \in \ell_{1}$. The map of interest is the map between $w$ to $z$, denoted by $T_{z w}$ :

$$
T_{z w}=G_{11}+G_{12} K\left(I-G_{22} K\right)^{-1} G_{21}
$$

This particular mapping captures the performance objectives. If $w \in \ell_{\infty}$ with $\|w\|_{\infty} \leq 1$ but other than that is arbitrary, then the nominal performance problem is defined as:

$$
\inf _{\text {stabilizing }}\left(\sup _{w}\left\|T_{z w} w\right\|_{\infty}\right)=\inf _{K \text { stabilizing }}\left\|T_{z w}\right\|_{1}
$$

\subsection{Controller Parameterization}

There are several ways for arriving to the parameterization of all stabilizing controllers, one of which is via coprime factorization $[4,6,5]$. In the 2 input 2-output set-up, the stabilizability of the system $G$ is equivalent to the stabilizability of $G_{22}$. Let $G_{22}$ have the bi-coprime factorization with $G_{22}=N M^{-1}=\tilde{M}^{-1} \tilde{N}$ :

$$
\left[\begin{array}{cc}
\tilde{X} & -\tilde{Y} \\
-\tilde{N} & \tilde{M}
\end{array}\right]\left[\begin{array}{cc}
M & Y \\
N & X
\end{array}\right]=I
$$


where all quantities above are stable. All stabilizing controllers are then parametrized as:

$$
K=(Y-M Q)(X-N Q)^{-1}=(\tilde{X}-Q \bar{N})^{-1}(\tilde{Y}-Q \tilde{M}) \quad Q \text { stable. }
$$

This parameterization has two major advantages: the first is that it furnishes the space of all stabilizing controllers (including time-varying and nonlinear) in terms of one parameter in a vector space, eventhough the space of all stabilizing controllers is not in itself a vector space. The second advantage, which is a great surprise, is that it transforms the complicated mapping $T_{z w}$ which is a nonlinear expression in the controller, to an affine linear function in the parameter $Q$. By simple manipulations, $T_{z w}$ is given by:

$$
T_{z w}=T_{1}-T_{2} Q T_{3}
$$

where

$$
\begin{gathered}
T_{1}=G_{11}+G_{12} M \tilde{Y} G_{21} \\
T_{2}=G_{12} M \\
T_{3}=\tilde{M} G_{21} .
\end{gathered}
$$

Define the feasible space $\mathcal{S}$ as follows:

$$
\mathcal{S}=\left\{R \in \ell_{1} \mid R=T_{2} Q T_{3}, Q \in \ell_{1}\right\}
$$

The $\ell_{1}$ optimal control problem is defined as follows:

$$
\inf _{R \in \mathcal{S}}\left\|T_{1}-R\right\|_{1}
$$

\section{Examples of Nominal Performace Objectives}

In this section, a few examples are presented to illustrate this formulation. These examples will show in a very simple way the advantages of using the $\ell_{\infty}$-norm on signals to reflect realistic time-domain specifications. 


\subsection{Disturbance Rejection}

In many real world applications, output disturbance and/or noise is persistent, i.e. continues acting on the system as long as the system is in operation. This implies that such inputs have infinite energy, and thus one cannot model them as "bounded-energy signals". Also, disturbances can be correlated with the inputs to the plant in a nonlinear fashion that makes it difficult to get accurate information about its statistics. Nevertheless, one can get a good estimate on the maximum amplitude of such inputs. In general, we will assume that the disturbance is the output of a linear-time invariant filter subjected to signals of magnitude less than or equal to one, i.e

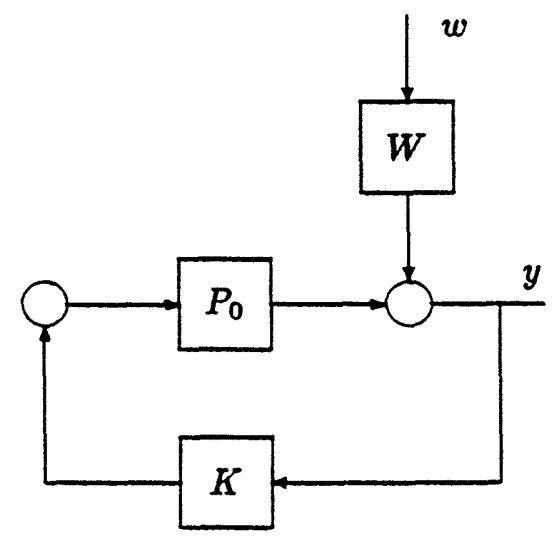

Figure 2: Disturbance Rejection Problem

$$
d=W w, \quad\|w\|_{\infty} \leq 1
$$

The disturbance rejection problem is defined as follows: Find a feedback controller that minimizes the maximum amplitude of the output over all possible disturbances. The two-input two-output system shown in Figure 2 is given by

$$
z=P_{0} u+W w
$$




$$
y=P_{0} u+W w
$$

\subsection{Command Following with Saturations}

The command following problem is equivalent to the disturbance rejection problem and is shown in Figure 3. In here, we will show how to pose this problem, in the presence of saturation nonlinearities at the input of the plant, as an $\ell_{1}$-optimal control problem. Define the function

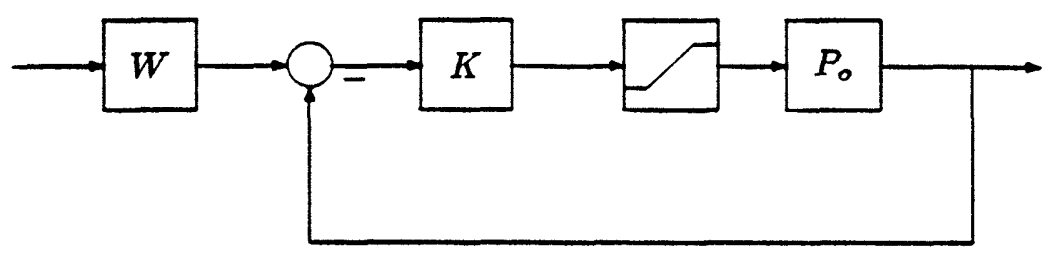

Figure 3: Command Following with Input Saturation

$$
\operatorname{Sat}(u)= \begin{cases}u & |u| \leq U_{\max } \\ U_{\max } & |u| \geq U_{\max }\end{cases}
$$

Let the plant be described as

$$
P_{u}=P_{0} S a t(u)
$$

where $P_{0}$ is $L T I$. Let the commands be modeled as

$$
r=W w,\|w\|_{\infty} \leq 1
$$

The objective is to find a controller $K$ such that $y$ follows $r$ uniformly in time. Keeping in mind the saturation function, it is clear that the allowable inputs have to have $\|u\|_{\infty} \leq U_{\max }$. Let $\gamma$ be a performance level desired, and define

$$
z=\left[\begin{array}{l}
\frac{1}{\gamma}(y-r) \\
\frac{1}{U_{\max }} u
\end{array}\right]
$$


with

$$
y=P_{0} u
$$

The problem is equivalent to finding a controller such that

$$
\left(\sup _{w}\|z\|_{\infty}\right)<1
$$

which is an $\ell_{1}$-optimal control problem.

Comment It is known to many that for such a problem, one will introduce some nonlinear function to minimize the effects of saturations on the plant. The formulation of this problem as a $\ell_{1}$ minimization problem is an example of the usage of this theory to highlight the fundamental limitations of linear controller design.

\subsection{Robust Stability}

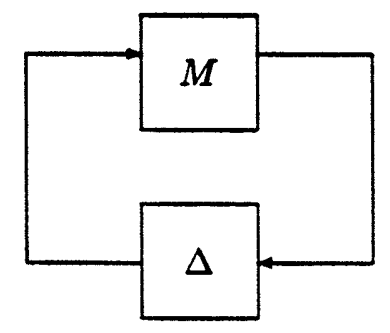

Figure 4: Stability Robustness Problem

Underlying most of the stability robustness results is the small gain theorem. Basically the theorem guarantees the stability of a feedback system consisting of an interconnection of two stable systems as in Figure 4 if the product of their gains is less than unity. This theorem is quite general and applies to nonlinear time-varying systems with any notion of $\ell_{p}$-stability. The small gain condition is in general not necessary for stability, however, it can be necessary if one of the systems in the feedback was arbitrary. Most of these results were previously treated in the literature for the case of $\ell_{2}$-stability 
[14], but were not studied in the case of $\ell_{\infty}$-stability. The next theorem is a surprising result in this sense and is due to Dahleh and Ohta [7].

Theorem 1 Let $M$ be a linear time-invariant system and $\Delta$ be a linear (possibly time-varying) stable system. The closed loop system shown in Figure 4 is $\ell_{\infty}$-stable for all $\Delta$ with gain $\sup _{\|f\|_{\infty} \leq 1}\|\Delta f\|_{\infty}<1$ if and only if $\|M\|_{1} \leq 1$.

The theorem implies the following: if $\|M\|_{1}>1$, then there exists a stable time-varying perturbation with gain less than unity such that the closed loop system is unstable. It is important that the perturbations $\Delta$ are allowed to be time-varying, otherwise the theorem may not be satisfied.

Example 1 Let

$$
\hat{M}(\lambda)=0.9 \frac{\lambda-0.5}{1-.5 \lambda} .
$$

The $\ell_{1}$ norm of $M$ is equal to 1.35 and the $H_{\infty}$ norm is equal to 0.9. Any destabilizing linear time-invariant perturbation satisfies:

$$
\|\Delta\|_{1} \geq\|\Delta\|_{\infty} \geq \frac{1}{0.9}>1
$$

However, a time-varying perturbation exists with a gain smaller than one.

As a consequence of the small gain theorem, it is possible to provide stability robustness conditions for some classes of perturbed systems.

\subsection{Unstructured Multiplicative Perturbations}

Consider the case where the system has input uncertainty in a multiplicative form as in Figure 5, i.e.

$$
\Omega=\left\{P \mid P=P_{0}\left(I+W_{1} \Delta W_{2}\right), \Delta \text { is time-varying with }\|\Delta\|<1\right\} .
$$

If a controller is designed to stabilize $P_{0}$, under what conditions will it stabilize the whole set $\Omega$ ? By simple manipulations of the closed loop system, the problem is equivalent to the stability robustness of the feedback system in Figure 4, with $M=W_{2} P_{0} K\left(I-P_{0} K\right)^{-1} W_{1}$. A necessary and sufficient 


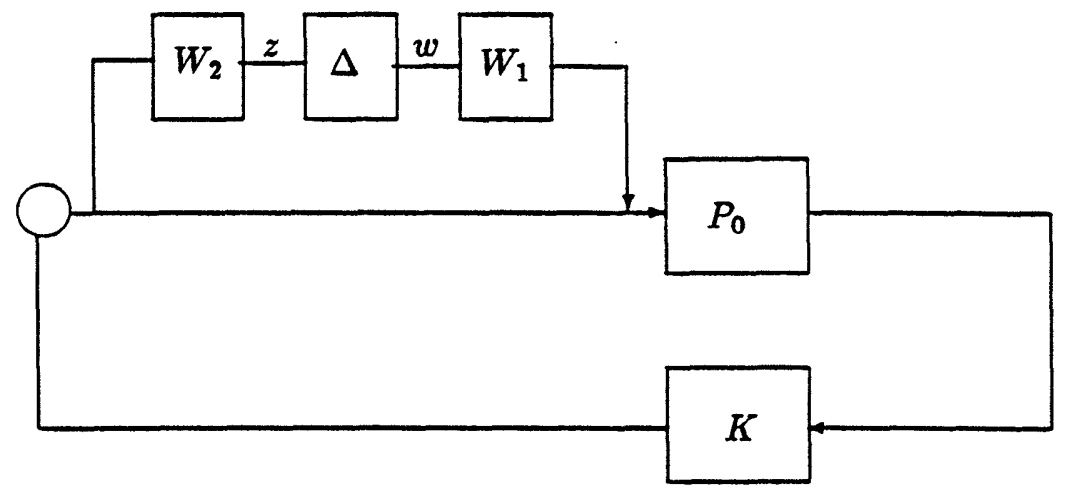

Figure 5: Multiplicative Perturbations

condition for robust stability is then given by $\|M\|_{1} \leq 1$. The resulting 2-input 2-output description is given by:

$$
\begin{gathered}
y=P_{0} u+P_{0} W_{1} w \\
z=W_{2} u
\end{gathered}
$$

\subsection{Robustness in the Presence of Stable Coprime Factor Perturbations}

Let $P_{0}$ be a linear time invariant, finite dimensional plant. As usual, $P_{0}=$ $G_{22}$. The graph of $P_{0}$ over the space $\ell_{q}$ is given by [5]:

$$
G^{q}\left(P_{0}\right)=G_{P_{0}} \ell_{q} \text { where } G_{P_{0}}=\left[\begin{array}{c}
M \\
N
\end{array}\right]
$$

Define the following class of plants as in Figure 6:

$$
\Omega_{q}=\left\{P \mid G_{P}=\left[\begin{array}{c}
M+\Delta_{1} \\
N+\Delta_{2}
\end{array}\right] \text { and }\left\|\left[\begin{array}{c}
\Delta_{1} \\
\Delta_{2}
\end{array}\right]\right\| \leq 1\right\}
$$

where $\Delta_{i}$ 's are $\ell_{q}$-stable linear time-varying systems, and the norm is the induced $\ell_{q}$ norm. The next theorem gives a necessary and sufficient condition for a controller that stabilizes $P_{0}$ to stabilize all $P \in \Omega_{\infty}$. A similar result in the case of $P \in \Omega_{2}$ was proved in [8]. It is evident that $\Omega_{\infty}$ contains time varying plants which will be essential for the proof of the next theorem capturing the stability robustness conditions [9]: 


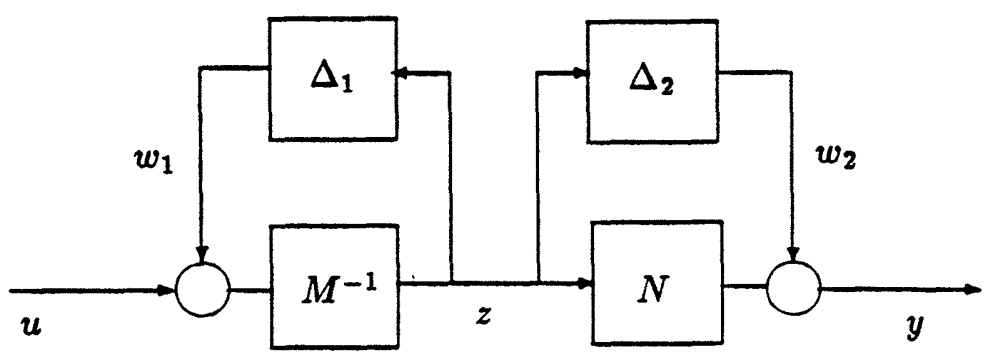

Figure 6: Coprime Factor Perturbations

Theorem 2 If $K$ stabilizes $P_{0}$, then $K$ stabilizes all $P \in \Omega_{\infty}$ if and only if

$$
\|[\tilde{X}-Q \tilde{N}-\tilde{Y}+Q \tilde{M}]\|_{1} \leq 1 .
$$

It is interesting to note that this class of perturbations is a more natural one in the case of unstable plants. It amounts to perturbing the graph of the operator, rather than the operator directly. This class of perturbations allow unstable perturbations and can result in changing the locations and the number of the unstable poles of the system. A description of the coprime perturbations of a system can be derived in a natural way from parameteric identification techniques in which a fixed order polynomial is identified for both $M, N$. The incorporation of such robustness results in adaptive controllers has proved quite effective as shown in [10,11].

If the above condition is not satisfied, then the theorem asserts that there exists an admissible time-varying plant which the controller does not stabilize. The 2-input 2-output description that results in the above criterion is given by:

$$
\begin{gathered}
y=P_{0} w_{1}+w_{2}+P_{0} u \\
z=M^{-1} w_{1}+M^{-1} u
\end{gathered}
$$

Comment There is a subtle difference between the construction of the plant which is destabilized by the controller in this case and the plant in the case of multiplicative perturbations. In the multiplicative perturbation case, if the 
stability robustness condition is not satisfied, the constructed destabilizing perturbation results in a plant with an internal unstable cancellation, thus cannot be stabilized by any controller. In the case of coprime factor perturbations, such plants can be ruled out from the set, and the destabilizing perturbation results in a stabilizable plant, however, not stabilized with the specific controller used [9].

\subsection{How big is this class of perturbation?}

As mentioned earlier, the small gain condition is applicable for general nonlinear time-varying systems, and hence tends to be a conservative condition for stability robustness. Nevertheless, it is a powerful tool for representing realistic classes of uncertainty such as unmodelled dynamics, ignored nonlinearities, time delays and so on.

Since the approach presented above is the same for the $H_{\infty}$ problem, it is worthwhile comparing the class of perturbations that have gain less than unity over $\ell_{2}$ with the class of perturbations that have gain less than unity over $\ell_{\infty}$. If the perturbations are restricted to time-invariant ones, the $\ell_{\infty}$-stable perturbations with gain less than unity lie inside the unit ball of $\ell_{2}$-stable perturbations (for the multivariable case, the unit ball will be scaled by a constant). This follows directly from the norm inequality between $\ell_{1}$ and $H_{\infty}$. If the perturbations are allowed to be time-varying, then the two sets are not comparable. Earlier, an example was presented that shows that the $H_{\infty}$ ball is larger than the $\ell_{1}$ ball. The next example furnishes a time varying operator which is $\ell_{\infty}$-stable but not $\ell_{2}$ stable.

Example 2 Define $\Delta$ as follows:

$$
(\Delta f)(k)=f(0)
$$

Clearly, this operator is $\ell_{\infty}$ stable but not $\ell_{2}$ stable.

\subsection{Duality between Stability and Performance}

In the previous examples, each of the robust stabilization problems was shown to be equivalent to some performance problem where a fictitious dis- 
turbance is injected at the output of the perturbation, and an error is measured at the input of the perturbation. So the transfer function to be minimized is simply the function seen by the perturbations. This says that robust stability is equivalent to some nominal performance problem. In the next section, the dual of this idea will be used: Performance will be equivalent to a robust stability problem in the presence of some fictitious perturbation. This will make the derivation of robust performance conditions a tractable problem.

\section{A Unified Approach for Stability and Perfor- mance Robustness}

In this section, it is shown that a general class of plant uncertainty can be described by linear fractional transformations. The robust performance problem will be posed as a robust stability problem in the presence of structured perturbations.

\subsection{Linear Fractional Transformation}

Given a $2 \times 2$ Block matrix transfer function $G$, we can define linear fractional transformations as

$$
\begin{aligned}
& F_{\ell}(G, K)=G_{11}+G_{12} K\left(I-G_{22} K\right)^{-1} G_{21} \\
& F_{u}(G, \Delta)=G_{22}+G_{21} \Delta\left(I-G_{11} \Delta\right)^{-1} G_{12}
\end{aligned}
$$

Consider the $3 \times 3$ system matrix $G$ shown in Figure 7 mapping

$$
\left(\begin{array}{c}
v \\
w \\
u
\end{array}\right) \rightarrow\left(\begin{array}{c}
r \\
z \\
y
\end{array}\right)
$$

where $v=\Delta r, \Delta \in \mathcal{D}, u=K y . w$ and $z$ denote the exogenous inputs and regulated outputs respectively, and $\mathcal{D}$ denotes the set of admissible perturbations. 


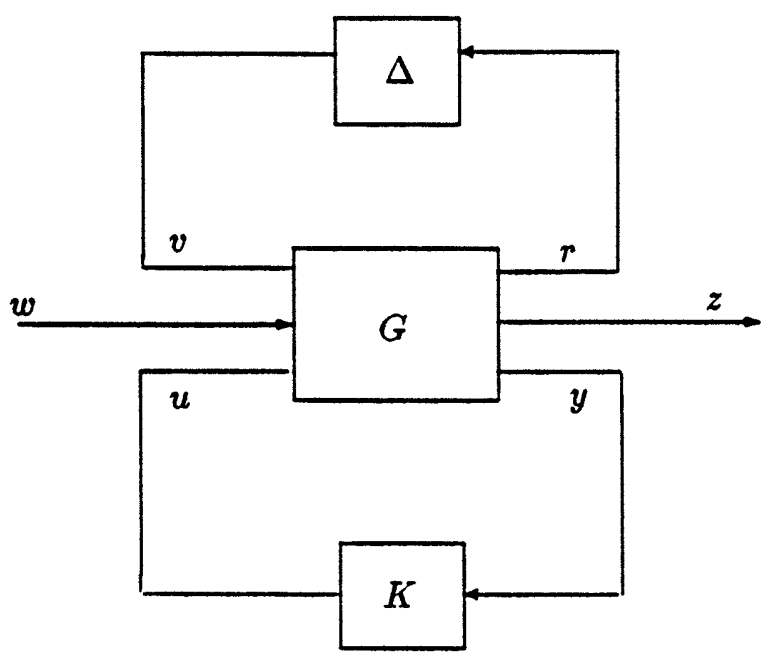

Figure 7: Perturbations Through LFT

$F_{\ell}(G, K)$ is the transfer matrix mapping

$$
\left(\begin{array}{c}
v \\
w
\end{array}\right) \rightarrow\left(\begin{array}{c}
r \\
z
\end{array}\right)
$$

with $u=K y . F_{\ell}(G, K)$ is the lower linear fractional transformation corresponding to $G$ partitioned conformally with

$$
\left(\begin{array}{c}
v \\
w \\
\cdots \\
u
\end{array}\right) \rightarrow\left(\begin{array}{c}
r \\
z \\
\cdots \\
y
\end{array}\right)
$$

$F_{u}(G, \Delta)$ is the transfer matrix mapping

$$
\left(\begin{array}{l}
w \\
u
\end{array}\right) \longrightarrow\left(\begin{array}{l}
z \\
y
\end{array}\right)
$$

with $v=\Delta r . F_{u}(G, \Delta)$ is the upper linear fractional transformation corresponding to $G$ partitioned conformally with

$$
\left(\begin{array}{c}
v \\
\cdots \\
w \\
u
\end{array}\right) \rightarrow\left(\begin{array}{c}
r \\
\cdots \\
z \\
y
\end{array}\right)
$$


Consider the class of plants $\Omega(G, \mathcal{D})$ in Figure 7 described as:

$$
\Omega(G, \mathcal{D})=\left\{P \mid P=F_{u}(G, \Delta) \text { for some } \Delta \in \mathcal{D}\right\}
$$

It will be shown later on that this represent a wide class of plant uncertainty.

\subsection{A General Class of Structured Uncertainty}

We now formally set up the stability and performance robustness problem for a class of structured uncertainty. The configuration we shall use in the setup of the robustness problem is shown in Figure 7 . The $3 \times 3$ system matrix $G$ represents the particular structure of interconnection of the nominal plant and the perturbations $\Delta$, and is therefore linear, time-invariant, and stable. The perturbation $\Delta$ has the form

$$
\Delta=\left(\begin{array}{cccc}
\Delta_{1} & 0 & \ldots & 0 \\
0 & \Delta_{2} & & \vdots \\
\vdots & & \ddots & 0 \\
0 & \ldots & 0 & \Delta_{n}
\end{array}\right)
$$

Each $\Delta_{i}$ represents the perturbation between two points in the system, and has norm less than or equal to one. Of course there is no loss of generality in assuming that the chosen bound on the norms of each of the $\Delta_{i}$ 's is one, since any other set of numbers could be absorbed in $G$. We will restrict the $\Delta_{i}$ 's to be strictly causal in order to guarantee the well posedness of the system. This is not a serious restriction and can be removed if it is known that the perturbation/nominal system connection is well-posed. Accordingly we can define the classes of perturbations to which the $\Delta_{i}^{\prime} s$ belong. Assuming the perturbations enter at $n$ places, and that each has $p_{i}$ inputs and $q_{i}$ outputs we have

$$
\Delta_{i} \in \Delta\left(p_{i}, q_{i}\right)
$$

where $\Delta\left(p_{i}, q_{i}\right):=\left\{\Delta \in \mathcal{L}_{T V}^{p_{i} \times q_{i}} \mid \Delta\right.$ is strictly causal and $\left.\|\Delta\| \leq 1\right\}$

Note that $\Delta_{i}$ is not dependent in any way on $\Delta_{j}$ when $j \neq i$. The only restriction is that $\Delta_{i}$ belongs to $\Delta\left(p_{i}, q_{i}\right)$ for each $i$. Next let $p=\sum_{i} p_{i}$, 
and $q=\sum_{i} q_{i}$. By $\mathcal{D}\left[\left(p_{1}, q_{1}\right) ; \cdots ;\left(p_{n}, q_{n}\right)\right]$ we mean the set of all operators mapping $\ell_{\infty}^{q}$ to $\ell_{\infty}^{p}$ of the form:

$$
\Delta=\left(\begin{array}{cccc}
\Delta_{1} & 0 & \ldots & 0 \\
0 & \Delta_{2} & & \vdots \\
\vdots & & \ddots & 0 \\
0 & \ldots & 0 & \Delta_{n}
\end{array}\right)
$$

where $\Delta_{i}$ belongs to $\Delta\left(p_{i}, q_{i}\right)$. When the pairs $\left(p_{i}, q_{i}\right)$ are known, they will be dropped from the notation and $\mathcal{D}$ will be understood to mean the above set. We will say the system in Figure 7 achieves robust stability if the system is stable for all $\Delta \in \mathcal{D}\left[\left(p_{1}, q_{1}\right) ; \ldots ;\left(p_{n}, q_{n}\right)\right]$.

Stability Robustness Problem. Find necessary and sufficient conditions for the controller $K$ to stabilize the class of plants

$$
\left\{P\left|P=F_{u}(G, \Delta)\right| \Delta \in \mathcal{D}\right\}
$$

On the other hand, we will say the system in Figure 7 achieves robust performance if the system is stable, and the effect of the exogenous inputs $w$ on the regulated output $z$ is attenuated for all $\Delta \in \mathcal{D}\left[\left(p_{1}, q_{1}\right) ; \ldots ;\left(p_{n}, q_{n}\right)\right]$.

Performance Robustness Problem. Find necessary and sufficient conditions for the controller $K$ to robustly stabilize the class of plants

$$
\left\{P\left|P=F_{u}(G, \Delta)\right| \Delta \in \mathcal{D}\right\}
$$

and satisfy

$$
\left\|T_{z w}\right\|_{\mathcal{L}_{T V}}<1 \quad \forall \Delta \in \mathcal{D} .
$$

When the controller is connected, the closed loop system of the plant and controller is given by $F_{l}(G, K)$ and in the next section will be denoted by $M$. The perturbation $\Delta$ will be connected to $M$ as a feedback system.

\subsection{Example}

Consider the case where the system has both input uncertainty and output uncertainty as shown in Figure 8. Define new variables $v_{1}, v_{2}, r_{1}, r_{2}$, 


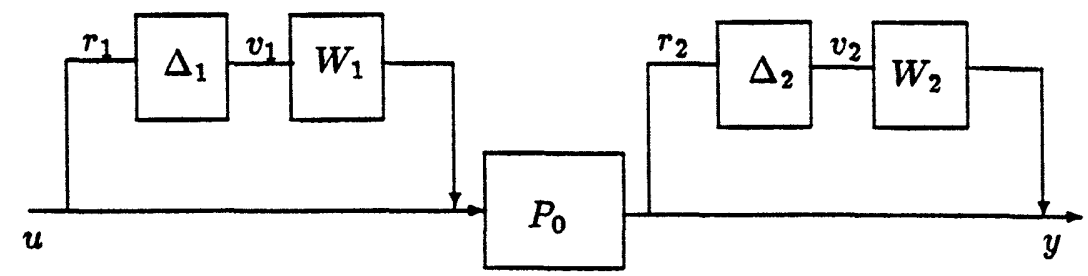

Figure 8: Input and Output Perturbations

where $v_{i}$ is the output of $\Delta_{i}$ and $r_{i}$ is the input to $\Delta_{i}$. Let $G$ be the transfer matrix from

$$
\left(\begin{array}{c}
v_{1} \\
v_{2} \\
u
\end{array}\right) \rightarrow\left(\begin{array}{c}
r_{1} \\
r_{2} \\
y
\end{array}\right)
$$

and is given by:

$$
G=\left(\begin{array}{ccc}
0 & 0 & I \\
P_{0} W_{1} & 0 & P_{0} \\
P_{0} W_{1} & W_{2} & P_{0}
\end{array}\right)
$$

Then, this class of uncertainty is represented as

$$
\Omega=\left\{P=F_{u}(G, \Delta) \mid \Delta \text { diagonal, }\|\Delta\|<1\right\} .
$$

\subsection{Performance Robustness Versus Stability Robustness}

In this section, we will establish a useful relationship between stability and performance robustness, that will be used later in the solution of our problem. This is achieved in the theorem below proved by Khammash and Pear. son [12]. It states that performance robustness in one system is equivalent to stability robustness in another one formed by adding a fictitious perturbation. A similar result has been shown to hold when the perturbations are linear time-invariant and when the 2-norm is used to characterize the perturbation class. The same proof does not apply here though, due to the assumed time-varying nature of the perturbations. The usefulness of this theorem stems from the fact that we can now concentrate on finding conditions for achieving stability robustness alone. Once we do, performance robustness comes for free. 


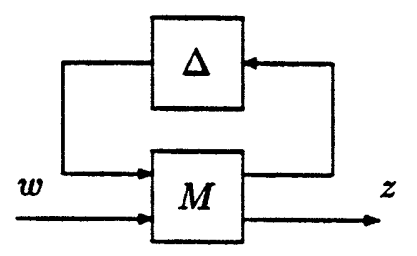

System I

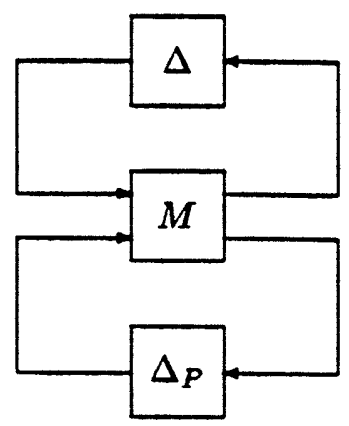

System II

Figure 9: Stability Robustness vs. Performance Robustness

Consider the two systems shown in Figure 9 , where $M \in \ell_{1}^{p \times q}$ and $\Delta_{i} \in \Delta\left(p_{i}, q_{i}\right)$. In system II, $w$ is an input vector of size $\tilde{p}$ and $z$ is an output vector of size $\tilde{q}$. In system $\mathrm{I}, \Delta_{p} \in \Delta(\tilde{p}, \tilde{q})$. It follows that $p=\tilde{p}+\sum_{i} p_{i}$ and $q=\tilde{q}+\sum_{i} q_{i}$. Subdivide $M$ in the following manner:

$$
M=\left(\begin{array}{ll}
\tilde{M}_{11} & \tilde{M}_{12} \\
\tilde{M}_{21} & \tilde{M}_{22}
\end{array}\right)
$$

where $\tilde{M}_{11} \in \ell_{1}{ }^{\tilde{p} \times \tilde{q}}$.

We now state the following theorem establishing the relation between System I and System II.

Theorem 3 The following four statements are equivalent:

i) System I achieves robust stability.

ii) $(I-M \bar{\Delta})^{-1}$ is $\ell_{\infty}-$ stable for all $\tilde{\Delta} \in \mathcal{D}\left[\left(p_{1}, q_{1}\right) ; \cdots ;\left(p_{n}, q_{n}\right) ;(\tilde{p}, \tilde{q})\right]$.

iii) $\left(I-\tilde{M}_{11} \Delta\right)^{-1}$ is $\ell_{\infty}$ - stable and $\left\|\tilde{M}_{22}+\tilde{M}_{21} \Delta\left(I-\tilde{M}_{11} \Delta\right)^{-1} \tilde{M}_{12}\right\|<$ 1 , for all $\Delta$ belonging to $\mathcal{D}\left[\left(p_{1}, q_{1}\right) ; \ldots ;\left(p_{n}, q_{n}\right)\right]$.

iv) System II achieves robust performance. 
The theorem basically says the following: to achieve robust performance, wrap a fictitious $\Delta_{p}$ mapping $z$ to $w$, and design a controller to achieve robust stability for the new system.

\section{Conditions for Stability Robustness}

In the previous sections, we have shown that the general Robust Performance problem is equivalent to a Robust Stability problem in the presence of structured perturbations. For any stabilizing controller, the conditions for Robust Stability are equivalent to necessary and sufficient conditions for the invertibility of $(I-\Delta M)$, where $M$ is a stable transfer function and $\Delta$ is block diagonal, i.e. $\Delta \in \mathcal{D}$. The next section will present these conditions in an exact form, which are due to Khammash and Pearson [12].

\subsection{Structured Small Gain Theorem}

Our Interest is to derive non-conservative conditions to guarantee the invertibility of $(I-\Delta M)$, where $\Delta \in \mathcal{D}$. From the small Gain Theorem, one condition is given by

$$
\|M\|_{1} \leq 1
$$

This condition is clearly conservative, i.e. if $\|M\|_{1}>1$ then the destabilizing perturbation may not have the diagonal structure shown. We can reduce the conservatism of the small gain theorem by introducing diagonal scalings that commute with the class of perturbations $\mathcal{D}$. In the sequel, we will treat the case of scalar $\Delta_{i}^{\prime} s$. The MIMO case follows in the same way.

Let $\mathcal{X}$ denote the class of all diagonal matrices with positive elements, $X \in \mathcal{X}$ if $X=\operatorname{diag}\left(x_{1}, \ldots, x_{n}\right)$ and $x_{i}>0$. It is evident that

$$
X \Delta X^{-1}=\Delta \quad \forall \Delta \in \mathcal{D}, X \in \mathcal{X}
$$

Hence, $(I-\Delta M)$ is given by $X^{-1}\left(I-\Delta X M X^{-1}\right) X$, and is stable if

$$
\left\|X M X^{-1}\right\|_{1} \leq 1
$$


In otherwords, if the above equation is valid for some $X$, then $(I-\Delta M)$ has a stable inverse. The least conservative choice will be

$$
\inf _{X \in \mathcal{X}}\left\|X M X^{-1}\right\|_{1} \leq 1
$$

While the above condition is less conservative, it is not clear how far it is from being necessary. The surprising fact is that the above condition is both necessary and sufficient, the proof of necessity will be highlighted below.

Denote by $\bar{M}$ the matrix $\left(\left\|m_{i j}\right\|\right)$, i.e. the matrix constructed by taking the norms of the $i j^{\text {th }}$ entry. It is straightforward to show that

$$
\|M\|=|\bar{M}|_{1}
$$

where $|A|_{1}=\max _{i} \sum_{j}\left|a_{i j}\right|$. The next theorem is a standard result from linear algebra known as the Perone-Frobenious theorem [13], and applies to all positive matrices. For simplicity, it is assumed that $\bar{M}$ has strictly positive elements.

Theorem 4 Given any matrix $M$, let $\bar{M}$ be defined as above, then the following hold:

i) $\inf _{X \in \mathcal{X}}\left\|X M X^{-1}\right\|_{1}=\lambda_{\max }(\bar{M})$.

ii) $\exists \tilde{x} \in \mathbb{R}^{n}, \tilde{x}_{i}>0$ such that $\bar{M} \tilde{x}=\lambda_{\max }(\bar{M}) \tilde{x}$.

iii) Define $\bar{X}=\operatorname{diag}\left(\tilde{x}_{1}, \ldots, \tilde{x}_{n}\right)$, then

$$
\inf _{X \in \mathcal{X}}\left\|X M X^{-1}\right\|_{1}=\left\|\tilde{X} M \tilde{X}^{-1}\right\|_{1} .
$$

It is interesting to note that $\tilde{X}$ gives the optimal scaling of both inputs and outputs to incorporate the directional information in the least conservative way. The theorem shows that the computation of $\bar{X}$ is straightforward for every fixed matrix $M$.

To summarize, a sufficient condition for robust stability in the presence of structured perturbations is given by:

$$
\inf _{X \in \mathcal{X}}\left\|X M X^{-1}\right\|_{1}=\lambda_{\max }(\bar{M}) \leq 1 .
$$


In the sequel, it is shown that the above condition is also necessary. To do that, we will present two key lemmas from which the proof will follow immediately.

Lemma 1 Given any $G \in \ell_{1}^{n \times n}$ such that $\left\|G_{i}\right\|_{1}=g_{i}>1$, where $G_{i}$ is the $i^{\text {th }}$ row of $G$. Then, $\exists f \in \ell_{\infty, e}^{n} \backslash \ell_{\infty}^{n}$ (unbounded), $n^{*}>0$ and $m>1$ such that

$$
\frac{\left\|P_{k-1} G_{i} f\right\|_{\infty}}{\left\|P_{k} f\right\|_{\infty}} \geq m \quad \forall k \geq n^{*} \forall i
$$

In words, this Lemma says that if the norm of each row of a matrix function $G$ is greater than 1 , then there exits an unbounded signal $f$ which gets amplified (in the $\ell_{\infty}$ sense) at each output channel after some fixed time $n^{*}$. This signal is then used to generate a time varying $\Delta$ that results in an unstable $(I-\Delta M)^{-1}$.

Lemma 2 Given $G_{i} \in \ell_{1}^{1 \times n}$ such that $\left\|G_{i}\right\|_{1}=g_{i}>1$. There exists a $\Delta_{i} \in \mathcal{L}_{T V}^{1 \times 1},\left\|\Delta_{i}\right\|<1$ s.t.

$$
f_{i}-\Delta_{i} G_{i} f \in \ell_{\infty}
$$

Equivalently

$$
(I-\Delta G) f \in \ell_{\infty} \text { with } \Delta=\operatorname{diag}\left(\Delta_{1}, \ldots, \Delta_{n}\right)
$$

The idea of the construction is quite straightforward: The signal $G f$ has components that are amplified in comparison to each component of $f$, after some time $n^{*}$. Thus it is possible to map back $(G f)_{i}$ to $f_{i}$ through a linear operator with norm less than 1 , for all time greater than $n^{*}$. With this, we are in a position to prove that condition (1) is both necessary and sufficient.

Theorem 5 Let $M \in \ell_{1}^{n \times n}, \Delta \in \mathcal{D}$. A necessary and sufficient condition for the inverse of $(I-\Delta M)$ to be $\ell_{\infty}$-stable is given by

$$
\inf _{\bar{X} \in \mathcal{X}}\left\|X M X^{-1}\right\|_{1}=\lambda_{\max }(\bar{M}) \leq 1
$$

where $\bar{M}$ is the matrix of norms of $M$. 
Proof. : Suppose $\lambda_{\max }(\bar{M})>1$, then there exists a positive vector $x \in \mathbb{R}^{n}$ s.t.

$$
\bar{M} x=\lambda_{\max } x
$$

Define $X=\operatorname{diag}\left(x_{1} \ldots, x_{n}\right)$, and $M_{i}$ be the $i^{\text {th }}$ row of $M$. We have

$$
\left\|M_{i} X\right\|=\left|\bar{M}_{i} x\right|=\lambda_{\max } x_{i}
$$

or equivalently

$$
\left\|\frac{1}{x_{i}} M_{i} X\right\|=\lambda_{\max }>1 \text {. }
$$

Let $G=X^{-1} M X$, then $G_{i}=\frac{1}{x_{i}} M_{i} X$ and $\left\|G_{i}\right\|>1$. By Lemma $2, \exists$ a $\Delta \in \mathcal{D}$ s.t $(I-\Delta G)^{-1}$ is not $\ell_{\infty}$-stable. However, $(1-\Delta G)^{-1}=X(I-$ $\Delta M)^{-1} X^{-1}$. Hence $(I-\Delta M)^{-1}$ is not $\ell_{\infty}$-stable.

Another interpretation of $X$ is simply the direction for the worst input, i.e. if we redefine the input $f$ in Lemma 1 as $\tilde{f}=X f$, then $M$ exerts the maximum amplification in that direction, the amplification is given by $\lambda_{\max }$.

Finally, it is worth noting that the problem of Stability Robustness and Performance Robustness of structured perturbations were originally formulated and developed by Doyle $[14,15]$ in what is now known as the $\mu$ theory. Similarities and contrasts between the $\mu$ theory and the method presented here are discussed in [16].

\section{Synthesis of $\ell_{1}$-Optimal Controllers}

The basic synthesis problem can be stated as follows: Find a controller $K$ such that

$$
\inf _{X \in \mathcal{X}}\left\|X M X^{-1}\right\|_{1}=\lambda_{\max }(\bar{M}) \leq 1
$$

Incorporating the $Q$ parameterization, the problem is equivalently stated as:

$$
\inf _{Q \in \ell_{1}} \inf _{X \in \mathcal{X}}\left\|X\left(T_{1}-T_{2} Q T_{3}\right) X^{-1}\right\|_{1} \text {. }
$$

Exact synthesis by simultaneously minimizing over $Q$ and $X$ is still an open problem. The best known method is an iterative method in which optimization in each of these variables is done independently. The infimization 
in $\mathcal{X}$ can be done exactly using Perone-Frobenious theorem for every fixed $Q$. Infimization over $Q$ is the main topic of the $\ell_{1}$ synthesis problem and will be described in the sequel. It is interesting to note that the problem is not jointly convex in both of these parameters and hence this scheme is guaranteed to converge only to local minima.

The problem of $\ell_{1}$ minimization was formulated by Vidyasagar in [17] as a minimax criterion that parallels the standard $H_{\infty}$ problem [18] and was solved by Dahleh and Pearson in $[19,20]$. Much work has been done on this problem afterwards, some of which can be found in $[9,16,21,22,23,24$, 25, 26]. The solution of Dahleh and Pearson hinged on the duality theory of minimum distance problems. In the sequel we will show that solutions can be obtained using only standard linear programming ideas, and the duality theory of Linear Programs.

\subsection{Characterization of the subspace $\mathcal{S}$}

Recall the $\ell_{1}$ minimization problem:

$$
\inf _{R \in \mathcal{S}}\left\|T_{1}-R\right\|_{1}
$$

where the subspace $\mathcal{S}$ is given by:

$$
\mathcal{S}=\left\{R \in \ell_{1} \mid R=T_{2} Q T_{3}, Q \in \ell_{1}\right\}
$$

The subspace $\mathcal{S}$ is in general limited by many factors such as zeros of $\hat{T}_{2}$ and $\hat{T}_{3}$ inside the unit disc, the rank of both of these systems and so on. There are two cases that arise, the first we term the good rank case in which the only constraints on $\mathcal{S}$ are the zeros of $\hat{T}_{2}, \hat{T}_{3}$ in the unit disc, and the second we term the bad rank case in which rank constraints also exists. One should bear in mind that the basic idea behind this characterization is the solvability of the equation

$$
\hat{R}=\hat{T}_{2} \hat{Q} \hat{T}_{3}
$$

for a stable $Q$. 


\subsection{Good Rank Case}

In here, it is assumed that $\hat{T}_{2}$ has full row rank $(=m)$ and $\hat{T}_{3}$ has full column rank $(=n)$. The reason for the term good rank is that the characterization of the subspace $S$ is obtained by finitely many equations and thus the optimization problem over $S$ will have an exact solution. For simplicity, it is assumed that $\hat{T}_{2}, \hat{T}_{3}$ have full rank for all $|\lambda|=1$.

The good rank problem usually arises in situation where one is interested in only one error function. An example of this is the sensitivity minimization problem discussed earlier. It is worthwhile noting that most of the interesting control problems violate these conditions, however, solutions for this class of problems offer a great insight into the solution of the general problem. In the next few sections, the presentation is essentially identical to [24], and is presented here for completeness.

Consider the Smith McMillan form decomposition of $\hat{T}_{2}, \hat{T}_{3}$ :

$$
\begin{aligned}
& \hat{T}_{2}=\hat{L}_{2} \hat{M}_{2} \hat{R}_{2} \\
& \hat{T}_{3}=\hat{L}_{3} \hat{M}_{3} \hat{R}_{3}
\end{aligned}
$$

where $L_{2}, R_{2}, L_{3}$, and $R_{3}$ are (polynomial) unimodular matrices and $M_{2}$, $M_{3}$ are rational matrices which have the familiar diagonal forms:

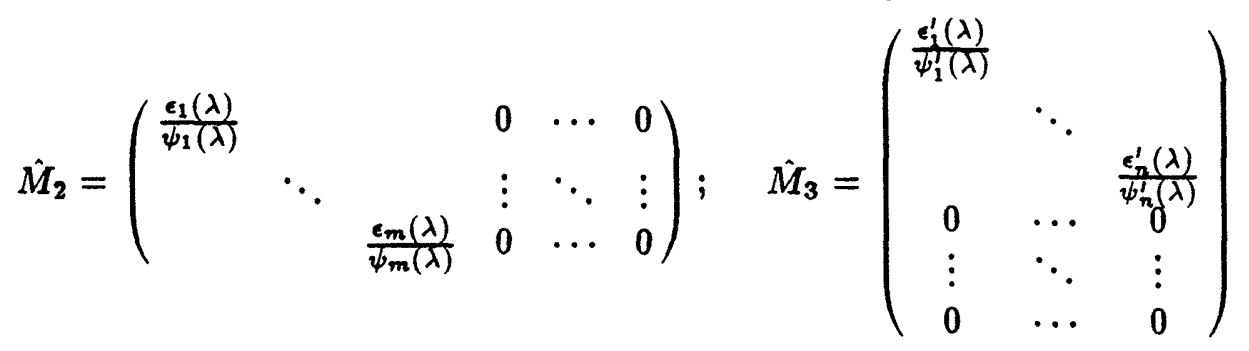

Let $\mathcal{Z}_{23}$ denote the set of all $\lambda \in \bar{D}$ which are zeros of either $\hat{T}_{2}$ or $\hat{T}_{3}$. Then for each $\lambda_{0} \in \mathcal{Z}_{23}$ we can define a non-decreasing sequence of nonnegative integers $\Sigma_{2}\left(\lambda_{0}\right)$ corresponding to the multiplicities with which the term $\left(\lambda-\lambda_{0}\right)$ appears on the diagonal of $\hat{M}_{2}$. That is:

$$
\Sigma_{2}\left(\lambda_{0}\right):=\left(\sigma_{2}^{i}\left(\lambda_{0}\right)\right)_{i=1}^{m}
$$

means:

$$
\frac{\epsilon_{i}(\lambda)}{\psi_{i}(\lambda)}=\left(\lambda-\lambda_{0}\right)^{\sigma_{2}^{i}\left(\lambda_{0}\right)} \hat{g}_{i}(\lambda) \quad i=1, \ldots, m
$$


where $\hat{g}_{i}(\lambda)$ has no poles or zeros at $\lambda=\lambda_{0}$. We can define similarly a set of sequences $\Sigma_{3}\left(\lambda_{0}\right)$ for each $\lambda_{0} \in \mathcal{Z}_{23}$ which correspond to the multiplicities of the $\lambda_{0}$ 's on the diagonal of $M_{3}$. A sequence $\Sigma_{3}\left(\lambda_{0}\right)$ is sometimes referred to as the sequence of structural indices of $\lambda_{0}$ in $\hat{T}_{2}$.

We can also define $m$ polynomial row vectors of dimension $m$ and $n$ polynomial column vectors of dimension $n$ as follows:

$$
\begin{array}{ll}
\hat{\alpha}_{i}(\lambda)=\left(\hat{L}_{2}^{-1}\right)_{i}(\lambda) & i=1, \ldots, m \\
\hat{\beta}_{j}(\lambda)=\left(\hat{R}_{3}^{-1}\right)^{j}(\lambda) & j=1, \ldots, n
\end{array}
$$

where subscript $i$ indicates the $i$-th row and superscript $j$ indicates the $j$-th column. Given the above definitions, a precise notion of interpolation which will be used in the sequel is presented.

Definition 1 Given $\hat{T}_{2}$ and $\hat{T}_{3}$ as above and $\hat{R} \in \mathbf{A}^{\mathbf{m} \times \mathbf{n}}$, we say $\hat{R}$ interpolates $\hat{T}_{2}$ (from the left) and $\hat{T}_{3}$ (from the right) if the following condition is satisfied: Given any zero $\lambda_{0} \in \mathcal{Z}_{23}$ of $\hat{T}_{2}$ and/or $\hat{T}_{3}$ with structural indices $\Sigma_{2}\left(\lambda_{0}\right)$ and $\Sigma_{3}\left(\lambda_{0}\right)$ in $\hat{T}_{2}$ and $\hat{T}_{3}$, respectively, we have for all $i \in\{1, \ldots, m\}$ and $j \in\{1, \ldots, n\}$ :

i). $\left(\hat{\alpha}_{i} \hat{R}\right)^{(k)}\left(\lambda_{0}\right)=0, \quad k=0, \ldots, \sigma_{2}^{i}-1$

ii). $\left(\hat{R} \hat{\beta}_{j}\right)^{(k)}\left(\lambda_{0}\right)=0, \quad k=0, \ldots, \sigma_{3}^{j}-1$

iii). $\sum_{l=0}^{k-\sigma_{3}^{j}} \sum_{r=0}^{\sigma_{3}^{j}-1}\left(\begin{array}{l}k \\ l\end{array}\right)\left(\begin{array}{c}k-l \\ r\end{array}\right)\left[\hat{\alpha}_{i}^{(l)} \hat{R}^{(k-l-r)} \hat{\beta}_{j}^{(r)}\right]\left(\lambda_{0}\right)=0, \quad k=\sigma_{3}^{j}, \ldots, \sigma_{2}^{i}+\sigma_{3}^{j}-1$

Or

$$
\sum_{l=0}^{k-\sigma_{2}^{i}} \sum_{r=0}^{\sigma_{2}^{i}-1}\left(\begin{array}{l}
k \\
l
\end{array}\right)\left(\begin{array}{c}
k-l \\
r
\end{array}\right)\left[\hat{\alpha}_{i}^{(l)} \hat{R}^{(k-l-r)} \hat{\beta}_{j}^{(r)}\right]\left(\lambda_{0}\right)=0, \quad k=\sigma_{2}^{i}, \ldots, \sigma_{3}^{j}+\sigma_{2}^{i}-1
$$
where the argument of $\sigma_{2}^{i}(\cdot)$ and $\sigma_{3}^{j}(\cdot)$ is understood to be $\lambda_{0}$ and superscript $(k)$ indicates the $k$-th derivative with respect to $z$.

Note that this condition simplifies greatly in the case of a zero $\lambda_{0}$ which is not common to $\hat{T}_{2}$ and $\hat{T}_{3}$; if it is a zero only of $\hat{T}_{2}$, for example, we have $\Sigma_{3}\left(\lambda_{0}\right)=(0)_{j=1}^{n}$ and parts $(i i)$ and $(i i i)$ are trivially satisfied for all $i$ and $j$. The next theorem gives a characterization of the subspace $\mathcal{S}$ in the good rank case. These conditions can be interpreted as a set of bounded linear functionals annihilating elements in $\mathcal{S}$. 
Theorem 6 Let $\hat{T}_{2}, \hat{T}_{3}$ satisfy the good rank assumptions, and $\hat{R} \in \mathbf{A}^{m \times n}$. Then there exists $\hat{Q} \in \mathbf{A}$ satisfying $\hat{R}=\hat{T}_{2} \hat{Q} \hat{T}_{3}$ if and only if $\hat{R}$ interpolates $\hat{T}_{2}$ and $\hat{T}_{3}$.

Proof. It is easily shown that for $\hat{R} \in \mathbf{A}^{\mathbf{m} \times \mathbf{n}}$ there exists $\hat{Q} \in \mathbf{A}^{\mathbf{m} \times \mathbf{n}}$ if and only if for all $\lambda_{0} \in \mathcal{Z}_{23}, i \in\{1, \ldots, m\}$ and $j \in\{1, \ldots, n\}$ we have:

$$
\left(\hat{\alpha}_{i} \hat{R} \hat{\beta}_{j}\right)^{(k)}\left(\lambda_{0}\right)=0, \quad k=0, \ldots, \sigma_{2}^{i}\left(\lambda_{0}\right)+\sigma_{3}^{j}\left(\lambda_{0}\right)-1
$$

The proof of the theorm follows from Weiner's theorm. The detailed proof can be found in [24].

The conditions captured in the above theorm can be viewed as forcing a collection of linear functionals to annihilate the subspace $\mathcal{S}$. The construction of such functionals is straightforward and follows similar to the SISO example shown in the sequel.

\subsection{The Bad rank Case}

In this case, $\hat{T}_{2}$ has full column rank $=n_{2}$ and $\hat{T}_{3}$ has full row rank $=n_{3}$. This situation occurs frequently in controller design since one is in general interested in many objectives for minimization. An example of the bad rank problem is the tracking example with saturations presented earlier.

In the sequel, it is assumed that there exists $n_{2}$ rows of $\hat{T}_{2}$ and $n_{3}$ columns of $\hat{T}_{3}$ which are linearly independent for all $\lambda$ on the unit circle. This assumption simplifies the exposition although it is not necessary. In general, it is enough to assume the above for one point on the unit circle [27]. Under this assumption, $\hat{T}_{2}$ and $\hat{T}_{3}$ can be written in the following form without loss of generality (possibly requiring the interchange of inputs and/or outputs):

$$
\begin{aligned}
& \hat{T}_{1}=\left(\begin{array}{l}
\hat{T}_{21} \\
\hat{T}_{22}
\end{array}\right) \\
& \hat{T}_{2}=\left(\begin{array}{ll}
\hat{T}_{31} & \hat{T}_{32}
\end{array}\right)
\end{aligned}
$$

where $\hat{T}_{21}$ has dimensions $n_{2} \times n_{2}$ and is invertible and $\hat{T}_{31}$ has dimensions $n_{3} \times n_{3}$ and is invertible. Moreover, $\hat{T}_{21}$ and $\hat{T}_{31}$ have no zeros on the unit circle. Thus $\hat{R}=\hat{T}_{2} \hat{Q} \hat{T}_{3}$ can be written:

$$
\hat{R}=\left(\begin{array}{l}
\hat{T}_{21} \\
\hat{T}_{22}
\end{array}\right) \hat{Q}\left(\begin{array}{ll}
\hat{T}_{31} & \hat{T}_{32}
\end{array}\right)=\left(\begin{array}{ll}
\hat{R}_{11} & \hat{R}_{12} \\
\hat{R}_{21} & \hat{R}_{22}
\end{array}\right)
$$


Notice that $\hat{T}_{21}$ and $\hat{T}_{31}$ define a good rank sub-problem, forcing $\hat{R}_{11}$ to interpolate their zeros. Nevertheless, this is not enough to characterize all the admissible $\hat{R}$ 's. The choice of $\hat{R}_{11}$ determines uniquely $\hat{Q}$. The rest of the elements in the $\hat{R}$ matrix have to be consistent with this solution, which in turn dictates a set of relations between the $\hat{R}_{i j}$. Define the following polynomial coprime factorizations:

$$
\begin{aligned}
& \hat{T}_{22} \hat{T}_{21}^{-1}=\hat{\tilde{D}}_{2}^{-1} \hat{\tilde{N}}_{2} \\
& \hat{T}_{31}^{-1} \hat{T}_{32}=\hat{N}_{3} \hat{D}_{3}^{-1}
\end{aligned}
$$

Using these definitions, we state the following result characterizing the feasible set $S$ for this case.

Theorem 7 Given $\hat{T}_{2}, \hat{T}_{3}$ with the assumption on the bad rank case, and $\hat{R} \in \mathbf{A}$, there exists $\hat{Q} \in \mathbf{A}$ satisfying $\hat{R}=\hat{T}_{2} \hat{Q} \hat{T}_{3}$ if and only if:

$$
\begin{aligned}
& \text { i). }\left(\begin{array}{ll}
-\hat{\hat{N}}_{2} & \hat{\tilde{D}}_{2}
\end{array}\right)\left(\begin{array}{ll}
\hat{R}_{11} & \hat{R}_{12} \\
\hat{R}_{21} & \hat{R}_{22}
\end{array}\right)=0 \\
& \text { ii). }\left(\begin{array}{ll}
\hat{R}_{11} & \hat{R}_{12}
\end{array}\right)\left(\begin{array}{c}
-\hat{N}_{3} \\
\hat{D}_{3}
\end{array}\right)=0 \\
& \text { iii). } \hat{R}_{11} \text { interpolates } \hat{T}_{21} \text { and } \hat{T}_{31} .
\end{aligned}
$$

The conditions shown in parts $i, i i$ are convolution constraints on the $\ell_{1}$ sequence. The interpolation condition in the last part can be tightened, since only the common zeros of $\hat{T}_{21}$ and $\hat{T}_{22}$ need to be interpolated.

The discussion above shows that the characterization of this subspace can be summerized by defining two operators:

$$
\mathcal{V}: \ell_{1}^{m \times n} \rightarrow \mathbb{R}^{\bullet}
$$

and

$$
\mathcal{C}: \ell_{1}^{m \times n} \rightarrow \ell_{1}^{r}
$$

where $s$ and $r$ are some integers. The first operator captures the interpolation constraints, and thus has a finite dimensional range, and the second captures the convolution constraints. These two operators can be constructed in a straightforward fashion, book-keeping being the only difficulty. To overcome 
this problem, it is helpful to think of $R$ as a vector rather than a matrix. To illustrate this, let the operator $\mathcal{W}$ be a map from $\ell_{1}^{m \times n}$ to $\ell_{1}^{m n}$ defined as follows:

$$
(\mathcal{W} R)(k)=\left(\begin{array}{c}
r_{11}(k) \\
\vdots \\
r_{m 1}(k) \\
r_{21}(k) \\
\vdots \\
r_{m n}(k)
\end{array}\right)
$$

The operator $\mathcal{W}$ is a one-to-one and onto operator, whose inverse is equal to its adjoint ( a fact used later). It simply re-arranges the variables in $R$. The conditions on $R$ presented in the above theorem can be written explicitly in terms of each component of $R$.

To construct the first operator $\mathcal{V}$, recall that each interpolation condition is interpreted as a bounded linear functional on $R$. By stacking up these functionals, the operator $\mathcal{V}$ is constructed. The following is an illustrative example.

Example 3 Suppose $\hat{T}_{21}$ and $\hat{T}_{31}$ are SISO and both have $N$ distinct zeros $a_{i}$ in the open unit disc. Then the matrix $\mathcal{V}$ is given by $\mathcal{V}=V_{\infty} \mathcal{W}$ where

$$
V_{\infty}=\left(\begin{array}{cccccccccc}
\operatorname{Re}\left(a_{i}^{0}\right) & 0 & 0 & 0 & \operatorname{Re}\left(a_{i}^{1}\right) & 0 & \ldots & \operatorname{Re}\left(a_{i}^{j}\right) & 0 & \ldots \\
\operatorname{Im}\left(a_{i}^{0}\right) & 0 & 0 & 0 & \operatorname{Im}\left(a_{i}^{1}\right) & 0 & \ldots & \operatorname{Im}\left(a_{i}^{j}\right) & 0 & \ldots
\end{array}\right)
$$

for $i=1, \ldots, N$ and $j=0,1,2, \ldots$.

For the second operator $\mathcal{C}$, recall that convolution can be interpreted as a multiplication by a block Toeplitz matrix, in this case with finite memory. By simple rearrangement, the operator is constructed with its image inside $\ell_{1}^{r}$. Hence $\mathcal{C}$ is given by $\mathcal{C}=\mathcal{T W}$ where $\mathcal{T}$ is a block lower triangular matrix. For a detailed example, see $[20,24]$. To illustrate the construction of the operator $\mathcal{T}$, consider the following example.

Example 4 consider the coprime-factor perturbation problem presented earlier for a SISO. The condition for stability robustness is given by [9]:

$$
\|[\tilde{V}-Q \tilde{N}-\tilde{U}+Q \bar{M}]\|_{1} \leq 1
$$


In this case, $T_{2}=1$ and $T_{3}=\left(\begin{array}{ll}\tilde{N} & -\tilde{M}\end{array}\right)$. Since $\tilde{M}^{-1} \tilde{N}=N M^{-1}$ with $N, M$ coprime, the conditions in the above theorem translate to

$$
\left(\begin{array}{ll}
R_{11} & R_{12}
\end{array}\right)\left(\begin{array}{c}
M \\
N
\end{array}\right)=0
$$

The matrix $\mathcal{T}$ is then given by (since $\mathcal{W}=I)$ :

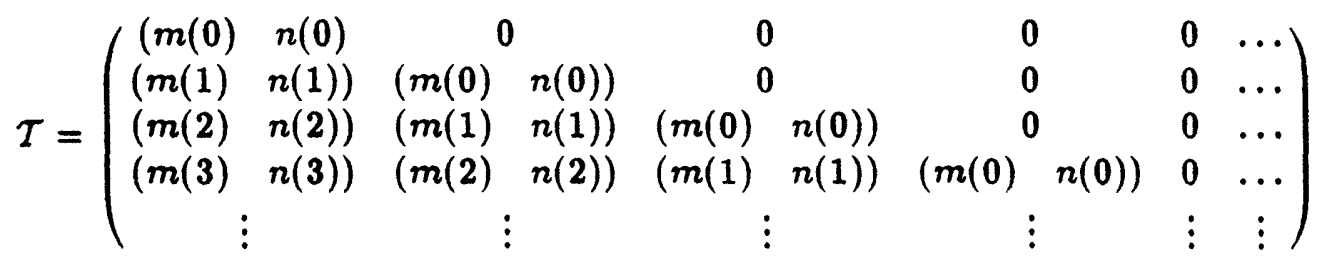

It is interesting to note that the operator $\mathcal{C}$ captures all the conditions and no interpolation conditions are needed. The conditions presented in the theorem can be redundant, and can be significantly reduced [27]. Generally, the operator $\mathcal{W} \neq I$ and so the operator $\mathcal{T}$ will not be exactly a Toeplitz matrix, although it will have a similar structure.

The subspace $\mathcal{S}$ is then the set of all elements $R \in \ell_{1}^{m \times n}$ such that $\mathcal{V} R=0$ and $C R=0$. Let $b_{1}=\mathcal{V} T_{1}$ and $b_{2}=C T_{1}$. The $\ell_{1}$ optimization problem can be restated as:

$$
\inf \|\Phi\|_{1} \quad \text { subject to } \mathcal{V} \Phi=b_{1}, \quad \mathcal{C} \Phi=b_{2}
$$

\section{Duality in Linear Programming}

It is well known that optimization problems minimizing the $\ell_{1}$-norm with linear constraints inside $\mathbb{R}^{n}$ are equivalent to linear programming (LP) problems. For this reason, it is natural to expect that the $\ell_{1}$ optimal control problem (OPT) is also equivalent to a linear programming problem. The difference is that such problems may have infinitely many variables and constraints, and thus only solvable by approximation. The duality theory in linear programming is primarily motivated by a desire to reduce the number of variables in a problem. It will be quite interesting if the infinite dimensional linear programs can be converted to finite-dimensional ones through some duality argument. In this section, it is shown that the good rank 
problems can be exactly transformed to finite-dimensional linear programs, however the bad rank problems may not. For the later, only approximate solutions can be obtained.

The following result is standard in linear programming and is known as the duality theorem: Let $x \in \mathbb{R}^{n}, A$ is an $m \times n$ matrix, $b$ is a $1 \times m$ vector and $c$ is a $1 \times n$ vector. The following two problems are equivalent:

$$
\begin{array}{cll}
\text { subject to } & \min c^{T}=\text { subject to } & \max b^{T} y \\
& A x \leq b & A^{T} y \geq c \\
& x \geq 0 & y \geq 0
\end{array}
$$

In words, a minimization problem with $n$ variables and $m$ constraints is equivalent to a maximization problem with $m$ variables and $n$ constraints. The constraint matrix of the second problem is the adjoint matrix (transpose) of the first. In a more mathematical terminology, $y$ is in the range of the adjoint matrix, hence is an element of the dual space of $\mathbb{R}^{m}$.

\subsection{The $\ell_{1}$ problem as a Linear Program}

In this section, only the SISO case is treated. The objective is simply to highlight the basic ideas involved in solving such problems not to present the most general solutions. Notice that in this case, the only constraints are due to $\mathcal{V}$ presented in the example above. An equivalent statement of OPT is given by:

$$
\min \mu
$$

subject to

$$
\begin{aligned}
& \|\Phi\|_{1} \leq \mu \\
& \mathcal{V} \Phi=b_{1} \\
& \Phi \in \ell_{1}
\end{aligned}
$$

To show the resemblance between OPT and standard linear programs, we can perform the following changes: $\Phi$ can be split into negative and positive components, $\Phi=\Phi^{1}-\Phi^{2}$ where $\phi^{1}(k), \phi^{2}(k) \geq 0$. This is a standard trick in linear programming that allows one to convert the $\ell_{1}$ norm to a linear objective function. By performing the minimization over $\Phi^{1}, \Phi^{2}$ the 
solution lies on the corners of the constraints, and hence at least one of the $\phi^{1}(k), \phi^{2}(k)$ will be zero for every $k$. This shows that $O P T$ is equivalent to: $\min \mu$

subject to

$$
\begin{aligned}
& \sum_{0}^{\infty} \phi^{1}(k)+\phi^{2}(k)-\mu \leq 0 \\
& \mathcal{V}\left(\Phi^{1}-\Phi^{2}\right)=b_{1} \\
& \phi^{1}(k), \phi^{2}(k) \geq 0
\end{aligned}
$$

Let $e^{T}=(1,1,1, \ldots)$. To put this problem in a matrix form, define the space $X=\ell_{1} \times \ell_{1} \times \mathbb{R}$ which can be viewed simply as an infinite sequence of variables. Define the matrix $\mathcal{A}$ decomposed conformally with $X$, whose range lie inside $\mathbb{R}^{2 N+1}$ as follows:

$$
\mathcal{A}=\left(\begin{array}{ccc}
e^{T} & e^{T} & -1 \\
\mathcal{V} & -\mathcal{V} & 0 \\
-\mathcal{V} & \mathcal{V} & 0
\end{array}\right)
$$

Also, define the vector $x \in X, b \in \mathbb{R}^{2 N+1}$ and the infinite vector $c$ (also decomposed conformally with $X$ ) as follows:

$$
x=\left(\begin{array}{c}
\Phi^{1} \\
\Phi^{2} \\
\mu
\end{array}\right) \quad b=\left(\begin{array}{c}
0 \\
b_{1} \\
-b_{1}
\end{array}\right) \quad c=\left(\begin{array}{l}
0 \\
0 \\
1
\end{array}\right)
$$

The matrix $\mathcal{A}$ has $2 N+1$ rows and an infinite number of columns. $O P T$ is equivalent to the following linear program:

$$
\min c^{T} x
$$

subject to

$$
\begin{aligned}
& \mathcal{A} x \leq b \\
& x \geq 0
\end{aligned}
$$

Using the LP duality theorem, $O P T$ is equivalent to another maximization problem in terms of a vector of dimension $2 N+1$. Let $\beta \in \mathbb{R}^{2 N+1}$ be decomposed as:

$$
\beta=\left(\begin{array}{c}
\beta_{1} \\
\beta_{2} \\
\beta_{3}
\end{array}\right) \quad \beta_{1} \in \mathbb{R}, \quad \beta_{2}, \beta_{3} \in \mathbb{R}^{N}
$$


The maximization problem is given by:

$$
\max \left(\beta_{2}-\beta_{3}\right)^{T} b_{1}
$$

subject to

$$
\begin{aligned}
& \beta_{1} e+\left(\beta_{2}-\beta_{3}\right)^{T} \mathcal{V} \geq 0 \\
& \beta_{1} e-\left(\beta_{2}-\beta_{3}\right)^{T} \mathcal{V} \geq 0 \\
& -\beta_{1} \geq-1 \\
& \beta_{1} \in \mathbb{R}, \quad \beta_{2}, \beta_{3} \in \mathbb{R}^{N}
\end{aligned}
$$

Finally, substituting $\alpha=\beta_{2}-\beta_{3}$, then $O P T$ is equivalent to the problem:

$$
\max \alpha^{T} b_{1}
$$

subject to

$$
\left|\alpha^{T} \mathcal{V}\right| \leq e
$$

where the inequality is taken pointwise. This problem is a finite dimensional linear program with infinitely many constraints. However, the matrix $\mathcal{V}$ has coefficients that decay exponentially, and one can show that only a finite number of constraints is needed to obtain an exact solution [19]. An explicit bound on the length of the finite dimensional problem can be derived.

Once a finite-dimensional dual problem has been determined and solved, the solution of the original problem (i.e. solution for $\Phi$ ) can be obtained directly from the linear program. The process of obtaining the primal solution is known as the alignment problem.

Note that in the above, an inequality formulation of the LP problem was chosen instead of the equality one. Although in the SISO this is not needed, for MIMO problems, the equivalent linear program will always have mixed constraints: equality and inequality constraints, and thus the above formulation is more direct. Similar results follow from the duality theory for Lagrange Multipliers and are reported in [16].

\subsection{The General Case}

In general, OPT may not be equivalent to a finite dimensional Linear Programming problem. In that case only approximate solutions can be obtained. 
We will not treat this case in here, however a good treatment is found in $[16,20,24,27]$. A straightforward way for solving the problem is to consider only finite length solutions for $\Phi$, and solve a finite dimensional LP as described earlier. As the length of the solution increases, suboptimal solutions to $O P T$ can be obtained. Duality theory can then be invoked to provide estimates for the distance between the actual minimum and the norm of the suboptimal solution.

\section{Conclusions}

This chapter presented an overview of the $\ell_{1}$ design methodology as a tool to synthesize controllers to achieve good performance in the presence of uncertainty. It was shown through prototype problems that this formulation is well suited for problems where Peak-to-Peak specifications and constraints are required. A general framework that allows incorporating stability robustness and performance robustness was presented from which computable, non-conservative conditions were derived. These conditions were shown to be equivalent to computing the spectral radius of some matrix, which was then simplified tremendously with the utilization of Perone-Frobenious theorem. The synthesis problem involved solving an $\ell_{1}$ optimization problem, which was shown to be intimately related to infinite Linear Programming problems.

The results presented in this chapter were only discrete-time results. The interest in discrete-time systems stems from the fact that in many practical situations, one is interested in designing digital controllers for a continuoustime plant. Such problems are known as sampled-data systems and has recently received a lot of attention in the literature. For hybrid systems with Peak-to-Peak specifications, it is shown in $[28,29,30]$ that these specifications can be met by solving a higher dimensional discrete-time $\ell_{1}$ problem. This in turn justifies the body of work on the pure discrete-time case.

There are many related results in the area of $\ell_{1}$ optimal control design. The sampled-data problem mentioned earlier is one area. A related area is 
the design of controllers for multi-rate sampled systems, or periodic systems [31]. Recently, a lot of attention has been given to the study of the structure of the $\ell_{1}$ controllers for the bad rank case searching for some separation structure similar to that of the $H_{2}$ and $H_{\infty}$ problems [32]. Exact solutions have been constructed for simple bad rank problems in [26, 27]. Also, it was shown that optimal solutions can require dynamic controllers eventhough all the states are available [22]. Other properties of optimal $\ell_{1}$ solutions are still under investigation [23]. Good demonstrations of the $\ell_{1}$ theory on practical problems can be found in [33].

In many practical problems, the external inputs include some fixed signals such as reference inputs. In such problems, the design methodology does not fall under the worst case paradigm, however still tractable using the above methods. In particular, controllers that will achieve performance objectives in terms of overshoot and settling time can be synthesized by solving linear programming problems and are discussed in details in $[34,35,21]$.

There are quite a few directions of research that are needed for the development of this methodology. To mention some of these, the problem of studying the structure of the $\ell_{1}$ controllers is quite important. Apart from providing a better insight into the design, the knowledge of the structure of the optimal controller can simplify the computations involved in a non-trivial fashion. Another research problem is concerned with model reduction: How can the order of the controller be reduced so that both stability and a level of performance is maintained. Also, the exact synthesis problem which involves a minimization of a spectral radius objective function is still an open problem. 


\section{References}

[1] C.A. Desoer and M. Vidyasagar, "Feedback Systems: Input-Output Properties," Academic Press, Inc, N.Y., 1975.

[2] J. C. Willems, "The Analysis of Feedback Systems." MIT Press, Cambridge, 1971.

[3] S.P. Boyd and J.C. Doyle, "Comparison of Peak and RMS Gains for Discrete-Time Systems," Syst. and Contr. Lett. 9, 1-6, (1987).

[4] B.A. Francis, "A Course in $H_{\infty}$ Control Theory, Springer-Verlag, 1987.

[5] M. Vidyasagar, "Control Systems Synthesis: A Factorization Approach," MIT press, 1985.

[6] D.C. Youla, H.A. Jabr, and J.J. Bongiorno, "Modern Wiener-Hopf Design of Optimal Controllers-part 2: The Multivariable Case," IEEETrans. A-C 21, 319-338, (1976).

[7] M.A. Dahleh and Y. Ohta, "A Necessary and Sufficient Condition for Robust BIBO Stability," Syst. and Contr. Lett. 11, 271-275, (1988).

[8] M. Vidyasagar and H. Kimura, "Robust Controllers for Uncertain Linear Multivariable Systems," Automatica 22, 85-94, (1986).

[9] M.A. Dahleh, "BIBO Stability Robustness for Coprime Factor Perturbations," to appear.

[10] M. Dahleh and M.A. Dahleh, "Optimal Rejection of Persistent and Bounded Disturbances: Continuity Properties and Adaptation," IEEE Trans. A-C 35, 687-696, (1990).

[11] P. Voulgaris M.A. Dahleh and Lena Valavani," Slowly-Varying Systems: $\ell^{\infty}$ to $\ell^{\infty}$ Performance and Implications to Adaptive Control," submitted. 
[12] M. Khammash and J. B. Pearson, "Performance Robustness of DiscreteTime Systems with Structured Uncertainty," IEEE Trans. A-C 36, 398412, (1991).

[13] R. Horn and C. Johnson, "Matrix Analysis," Cambridge University Press, 1985.

[14] J.C. Doyle and G. Stein, "Multivariable Feedback Design: Concepts for a Classical/Modern Synthesis," IEEE-Trans. A-C 26, 4-16, (1981).

[15] J. C. Doyle, "Analysis of Feedback Systems with Structured Uncertainty," IEEE Proceedings 128, 242-250, (1982).

[16] M.A. Dahleh and M.H. Khammash, "Stability and Performance Robustness in the Presence of Bounded But Unknown Inputs," to appear in Automatica special issue on robust control.

[17] M. Vidyasagar "Optimal Rejection of Persistent Bounded Disturbances," IEEE-Trans. A-C 31, 527-534, (1986).

[18] G. Zames, "Feedback and Optimal Sensitivity: Model Reference Transformations, Multiplicative Seminorms, and Approximate Inverses," IEEE-Trans. A-C 26, 301-320, (1981).

[19] M.A. Dahleh and J.B. Pearson, " $l$ " Optimal Feedback Controllers for MIMO Discrete-Time Systems," IEEE Trans. A.C 32, 314-322, (1987).

[20] M.A. Dahleh and J.B. Pearson, "Optimal Rejection of Persistent Disturbances, Robust Stability and Mixed Sensitivity Minimization," IEEE Trans. Automat. Contr. 33, 722-731, (1988).

[21] G. Deodhare and M. Vidyasasgar. "Some Results on $\ell_{1}-$ Optimality of Feedback Control Systems: The SISO Discrete-Time Case", IEEE Trans. A-C, 35, 1082-1085, (1990).

[22] I. Diaz-Bobillo and M.A. Dahleh, "State Feedback $\ell_{1}$ Optimal Controllers Can be Dynamic," LIDS, MIT, Report No. LIDS-P-2051, (1991). 
[23] I. Diaz-Bobillo and M.A. Dahleh, "On the Solution of the $\ell_{1}$ Optimal Multi-Block Problem,", MIT Report in preparation.

[24] J.S. McDonald and J.B. Pearson, " $\ell_{1}-$ Optimal Control of Multivariable Systems with Output Norm Constraints," Automatica 27, 317-329, (1991).

[25] M.A. Mendlovitz, "A Simple Solution to the $\ell_{1}$ Optimization Problem," Syst. and Contr. Lett. 12, 461-463, (1989).

[26] O.J. Staffans, "Mixed Sensitivity Minimization Problems with Rational $\ell_{1}$-optimal solutions", Journal of Optimization Theory and Applications 70, 173-189, (1991).

[27] O.J. Staffans, "On the Four-block Model Matching Problem in $\ell_{1}$," Helsinki University of Technology, Espoo, Report A289, (1990).

[28] B.A. Bamieh, M.A. Dahleh and J.B. Pearson, "Minimization of the $L^{\infty}$-Induced Norm for Sampled-Data Systems," submitted.

[29] G. Dullerud and B.A. Francis, " $\mathcal{L}^{1}$ Performance in Sampled-Data Systems," submitted.

[30] M.H. Khammash. "Necessary and Sufficient Conditions for the Robustness of Time-Varying Systems with Applications to Sampled-Data Systems," submitted.

[31] M.A. Dahleh, P. Voulgaris and L. Valavani, "Optimal and Robust Controllers for Periodic and Multi-Rate Systems," to appear.

[32] J.C. Doyle, K. Glover, P.P. Khargonekar and B.A. Francis, "State Space Solutions to Standard $\mathrm{H}_{2}$ and $H_{\infty}$ Control Problems," IEEE Trans. AC 34, 831-847, (1989).

[33] M.A. Dahleh and D. Richards, "Application of Modern Control Theory on a Model of the X-29 Aircraft," LIDS, MIT, report No. LIDS-P-1932 (1989). 
[34] S.P. Boyd and C.H. Barratt, "Linear Controller Design: Limits of Performance," Prentice Hall, N.J., 1991.

[35] M.A. Dahleh and J.B. Pearson. "Minimization of a Regulated Response to a Fixed Input," IEEE Trans. Automat. Contr. 33, 924-930,(1988). 